\title{
Influencia de las habilidades organizacionales de los alumnos de IX y X ciclo de la EAPIC-UAP, Filial Piura, durante el ciclo académico 2009-2A, en su nivel de desempeño laboral
}

\section{Influence of Organizational Skills for Students of IX and X Cycle EAPIC-UAP-Filial Piura, during the academic 2009-2A, in their Level Job Performance}

\author{
Marina Jovita Lam Lau*
}

http://dx.doi.org/10.21503/CienciayDesarrollo.2011.v13.06

\section{RESUMEN}

El presente estudio tiene por objetivo determinar el nivel de desempeño laboral que proyectarían los alumnos de IX y X ciclo de la Escuela de Ingeniería Civil - EAPIC-UAP (Filial Piura), ante las actuales exigencias organizacionales producidas por efecto de la globalización en nuestro país. Para la evaluación se consideraron tres aspectos: liderazgo, estilo de trabajo en equipo y estilo de manejo de conflictos. La metodología de la presente investigación es de tipo descriptivo-correlacional, corresponde a la realización de un proyecto de investigación y responde al nivel descriptivo-explorativo. Su enfoque principal es de tipo cuantitativo y el diseño es transversal, descriptivo y correlacional.

Luego de la toma de encuestas a los alumnos de IX y X ciclo de la EAPIC-UAP del ciclo académico 2009-2A, se obtuvo como resultado final que, a nivel general, el 100\% de ellos proyectarán un nivel medio de desempeño laboral, siendo importante destacar que a nivel individual un $14 \%$ de nuestros egresados proyectaría un alto nivel de desempeño laboral. Estos resultados generan buenas expectativas para la imagen de nuestra universidad, pero a su vez también exigen el compromiso de nuestra institución de establecer cursos complementarios que permitan mejorar el nivel laboral obtenido, lo que redundará en beneficio de nuestros jóvenes, nuestra universidad, sus familias y nuestro país.

Palabras clave: nivel de desempeño laboral, liderazgo, trabajo en equipo, manejo de conflictos.

\section{ABSTRACT}

The present study aims to determine the level of job performance that would project the students of IX and X Cycle of the Civil Engineering School - EAPIC-UAP (Filial Piura), to the current organizational requirements produced by the effect of globalization in our country. For the evaluation were considered three areas: Leadership, Style Teamwork and Conflict Management Style. The methodology of this research is descriptive and correlational, corresponds to the creation of a Research Project and reflects the explorative descriptive level. Its primary focus is a quantitative and its design is transversal, descriptive and correlational.

After making inquiries with students of IX and X Cycle of EAPIC-UAP of the Academic Cycle 2009$2 \mathrm{~A}$, the end result was that, in general, $100 \%$ of them projected an average level of job performance, still important to note that at the individual level $14 \%$ of our graduates plan a high level of job performance. These results produce great expectations for the image of our university, but in turn also require the commitment of our institution to set up courses to improve the working level obtained, which will benefit our youth, our university, their families and our country.

Key words: level of job performance, leadership, teamwork, conflict management

* Docente de Tecnología de los Materiales. Universidad Alas Peruanas. Ingeniera Civil y Maestra en Docencia Universitaria y Gestión Educativa. (e-mail: marinajovita@yahoo.es) 


\section{INTRODUCCIÓN}

La presente investigación, titulada Influencia de las habilidades organizacionales de los alumnos de IX y X ciclo de la EAPIC$U A P$, Filial Piura, durante el ciclo académico 2009-2A, en su nivel de desempeño laboral, tiene como objetivo principal determinar el nivel de desempeño laboral que proyectarían los alumnos de IX y X Ciclo de la Escuela Académico Profesional de Ingeniería Civil (EAPIC) de la Universidad Alas Peruanas (UAP), Filial Piura, frente a las actuales exigencias organizacionales producidas por efecto de la globalización en nuestro país.

Esta investigación resulta importante por ser la primera que ha sido dirigida a evaluar tres de las habilidades en comportamiento organizacional de los alumnos de la EAPICUAP (Filial Piura): liderazgo, estilo de trabajo en equipo y estilo de manejo de conflictos, teniendo en cuenta que actualmente a nivel internacional el comportamiento organizacional se considera como la base del éxito de toda empresa, y que nuestros futuros egresados formarán parte de organizaciones cuyo principal valor estará determinado por la competitividad, el alto nivel de desempeño y la búsqueda personal de elevados niveles de calidad de vida por parte de las personas que las conformen (Schermerhorn, Hunt y Osborn, 2004, 3).

La estructura de esta investigación comprende cinco (05) capítulos: Planteamiento teórico, Planteamiento del problema, Metodología, Presentación y análisis de resultados, y Conclusiones y recomendaciones. Adicionalmente incluye la Bibliografía y los Anexos (formatos de encuestas y resultados detallados de encuestas).

Esperamos que esta investigación sea un aporte valioso, por la información presentada, los instrumentos utilizados y su originalidad en el tema tratado, los mismos que buscan garantizar que nuestros futuros profesionales respondan a las demandas del proceso productivo y del mercado ocupacional actual (Farro, 2005, 320).

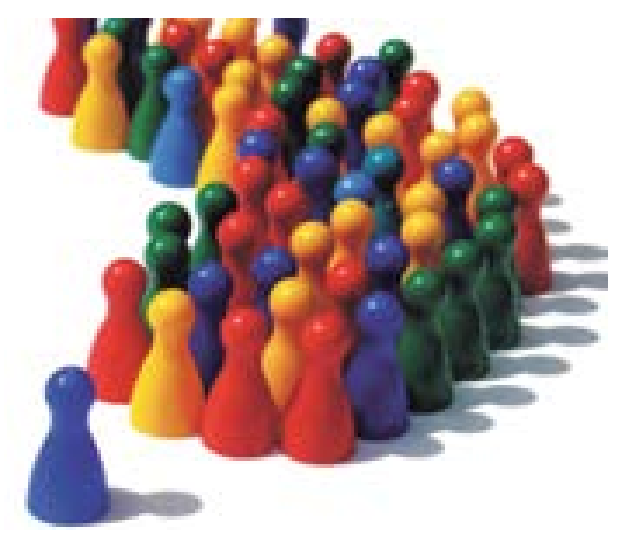

Figura 1. Liderazgo.



Figura 2. Trabajo en equipo.

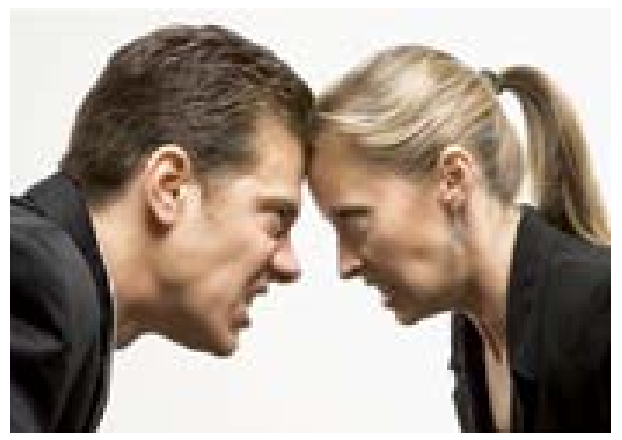

Figura 3. Manejo de conflictos.

\section{Justificación del estudio}

- En Piura, del 100\% de su PEA (Población Económicamente Activa), solo el 6\% está en el sector construcción, mientras que el 
$43,9 \%$ está en el sector Servicio y el 30,8\% en el sector comercio (INEI, 2008, versión digital, cap. 7, cuadro 7.14). Esto implica que nuestros futuros egresados se dedicarán a diversas actividades laborales y no netamente a las de su carrera profesional de origen, por lo que deberán estar preparados para ello, y es la Universidad la encargada de capacitar al futuro profesional a fin de que pueda incorporarse exitosamente al proceso productivo y al mundo laboral; en caso contrario, como institución educativa no tendrá calidad para el consumidor o usuario ni para la sociedad (Farro, 2005, 21).

- La Asamblea Nacional de Rectores, en su "Modelo de Autoevaluación con Fines de Mejora de las Carreras Universitarias" (ANR, 2007, 28 y 45), establece lo siguiente:

- "LaUniversidaddebefundamentar lacarrera profesional en función de la demanda social presente y futura, a nivel local, regional, nacional e internacional, y el perfil del egresado debe definir los conocimientos, las capacidades, las habilidades, las actitudes y competencias a adquirirse" durante su formación profesional.

- La universidad debe asegurar un mínimo de empleabilidad de sus egresados y contar con un número significativo de egresados que ocupen cargos de alta dirección.

Esto implica que toda institución de educación superior que se catalogue de calidad debe asumir la responsabilidad de autoevaluar sus planes curriculares y el estado laboral de sus egresados en forma periódica. Adicionalmente, es importante el seguimiento a los egresados que logran éxito al ocupar cargos de alta dirección.

Ambos aspectos, la autoevaluación continua y el seguimiento laboral de nuestros futuros egresados, motivan a la realización de la presente investigación, ya que de esta manera podremos determinar, mediante una proyección a futuro, cuál será su nivel de desempeño laboral, desde el enfoque de sus habilidades organizacionales.

- Del III Taller Internacional "Educando al Ingeniero del Siglo XXI" se desprende la necesidad de reformular la educación de los futuros ingenieros a fin de que no solo adquieran habilidades técnicas sino también complementarias a las de su carrera, lo que les garantizará contar con las herramientas necesarias para mantenerse activos de aquí a 40 años hacia delante (Hedberg, 2001).

- La EAPIC-UAP (a nivel nacional) establece en su perfil de la carrera que la escuela tiene "la finalidad de formar profesionales idóneos que coadyuven con el desarrollo integral del país y de la región en especial; que estén preparados para que su desempeño sea claro, con sentido de ética y responsabilidad social. Su sólida preparación deberá permitirles que se adapten rápido a los cambios de la técnica moderna y que puedan resolver con solvencia los problemas técnicos de su especialidad; con una formación integral que los capacite para convertirse en líderes que participan en la toma de decisiones" (Navarro, 2007).

Esto conlleva que el futuro ingeniero civil esté preparado a nivel general en los siguientes aspectos: planificación, organización, dirección y control (http:// www.gestionyadministracion.com/ empresas/concepto-de-gestion.html); lo que involucra la capacidad de trabajo en equipo, el liderazgo y la capacidad de manejo de conflictos por parte de nuestros alumnos.

- Nuestros futuros ingenieros civiles deben estar al tanto de las actuales características que se dan a nivel laboral. Investigaciones recientes han encontrado la denominada Generación $\mathrm{X}$ o de Trabajadores de cuello dorado (aquellos que han nacido entre 1965 y 1977), que poseen características laborales muy especiales: demandan mucho a sus compañías, desean 


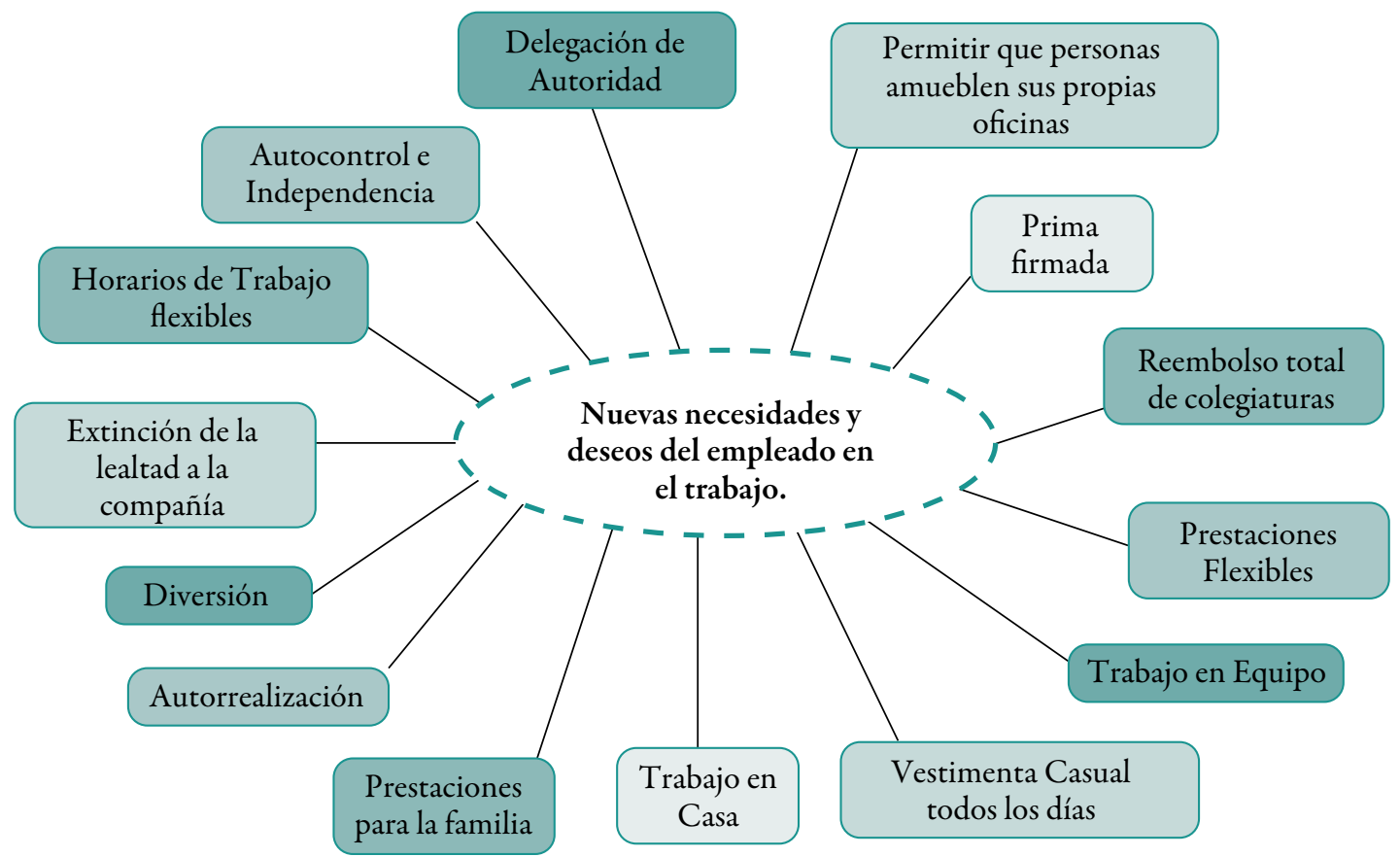

Figura 4. Evolución de la fuerza laboral: valores y preferencias de la Generación X o trabajadores de cuello dorado.

retos en sus puestos de trabajo y horarios de trabajo flexibles, trabajar en casa y trabajar en equipo, etc. (ver Figura 4).

\section{Objetivos del estudio}

\section{a) Objetivo general}

Determinar el nivel de desempeño laboral que proyectarían los alumnos de IX y X Ciclo de la EAPIC-UAP (Filial Piura) frente a las actuales exigencias organizacionales producidas por efecto de la globalización en nuestro país.

\section{b) Objetivos especificos}

- Describir los rasgos de liderazgo que presentan los alumnos de IX y X Ciclo de la EAPIC-UAP (Filial Piura).

- Determinar el estilo de trabajo en equipo que predomina en los alumnos de IX y X ciclo de la EAPIC-UAP (Filial Piura).

- Determinar el estilo de manejo de conflictos que predomina en los alumnos de IX y $\mathrm{X}$ ciclo de la EAPIC-UAP (Filial Piura).

\section{MATERIALES Y MÉTODOS}

Nivel, enfoque y diseño. El estudio es de nivel descriptivo-explorativo, su enfoque es de tipo cuantitativo y corresponde a un diseño transversal - descriptivo- correlacional.

Limitaciones. La investigación estuvo limitada al estudio de tres (03) habilidades organizacionales, las cuales fueron seleccionadas por la investigadora debido al interés personal de evaluar estos tres aspectos, por estar dentro de las más importantes del total de habilidades en comportamiento organizacional existentes: liderazgo, estilo de trabajo en equipo y estilo de manejo de conflictos.

Población. Estuvo conformada por los alumnos de IX y X ciclo de la Escuela AcadémicoProfesional de Ingeniería Civil de la Universidad Alas Peruanas-Filial Piura, del ciclo académico 2009-2A (setiembre 2009 a enero 2010). La muestra total fue de 22 alumnos. 


\section{RESULTADOS}

Tabla 1. Determinación del nivel de rasgos de liderazgo por alumno.

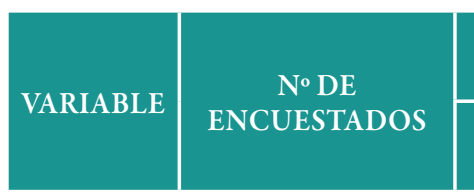

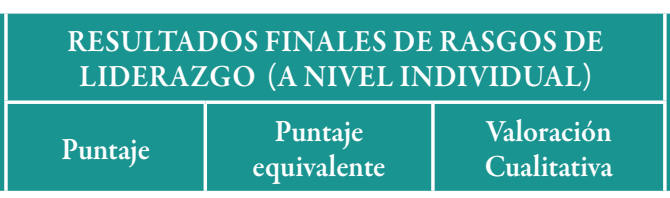

Encuestado No 1

Encuestado $\mathrm{N}^{\circ} 2$

Encuestado No 3

Encuestado No 4

Encuestado No 5

Encuestado $\mathrm{N}^{\circ} 6$

Encuestado No 7

Encuestado No 8

Encuestado No9

Encuestado No 10

Encuestado No 11

Encuestado No 12

Encuestado No 13

Encuestado No 14

Encuestado No $15 \quad 42$

Encuestado No $16 \quad 37$

Encuestado No $17 \quad 39$

Encuestado No $18 \quad 38$

Encuestado No 19

Encuestado No 20

Encuestado No 21

Encuestado No 22

32

14,2

16,4

16,9

11,6

15,6

15,6

38

17,3

44

33

13,6

36

36

14,7

16,0

38

16,0

16,3

$39 \quad 17,3$

17,8

10,7

16,4

17,3

16,9

37

16,4

35

41

15,6
16,2
15,6

$35 \quad 15,6$

Regular

Bueno

Bueno

Muy Bueno

Bueno

$\begin{array}{ll}\text { Bueno } & \text { autoritario / liberal } \\ \text { Bueno } & \text { autoritario / liberal }\end{array}$

\section{ESTILO DE LIDERAZGO PREDOMINANTE \\ PREDOMINANTE}

autoritario / liberal

Bueno autoritario / democràtico / liberal

democrático / liberal

No se identifica con ningún estilo

Bueno autoritario / democrático / liberal autoritario / democrático / liberal

No se identifica con ningún estilo

Bueno democrático / liberal

$$
\text { democrático }
$$

Bueno democrático

$\begin{array}{cc}\text { Bueno } & \text { autoritario / democrático / liberal } \\ \text { Bueno } & \text { autoritario / democrático / liberal } \\ \text { Muy Bueno } & \text { democrático }\end{array}$

Fuente: elaboración propia (resultados de encuesta a alumnos).

Tabla 2. Nivel de rasgos de liderazgo de alumnos de IX y X ciclo de la EAPIC-UAP-Filial Piura (a nivel individual).

\begin{tabular}{c|c|c|c|}
\hline $\begin{array}{c}\text { NIVEL DE RASGOS } \\
\text { DE LIDERAZGO }\end{array}$ & FRECUENCIA & $\begin{array}{c}\text { PORCENTAJE } \\
\text { PARCIAL }\end{array}$ & $\begin{array}{c}\text { PORCENTAJE } \\
\text { ACUMULADO }\end{array}$ \\
\hline Muy bueno & 3 & $14 \%$ & $14 \%$ \\
\hline Bueno & 18 & $82 \%$ & $96 \%$ \\
\hline Regular & 1 & $4 \%$ & $100 \%$ \\
\hline Bajo & 0 & $0 \%$ & $100 \%$ \\
\hline Total & 22 & $100 \%$ & \\
\hline
\end{tabular}

Fuente: elaboración propia (obtenida de tabla 1). 
Interpretación. De las tablas 1 y 2 tenemos que el $14 \%$ de los alumnos presenta muy buen nivel de rasgos de líderes, el $82 \%$ presenta buen nivel de rasgos de líderes, y el 5\% presenta un nivel regular.

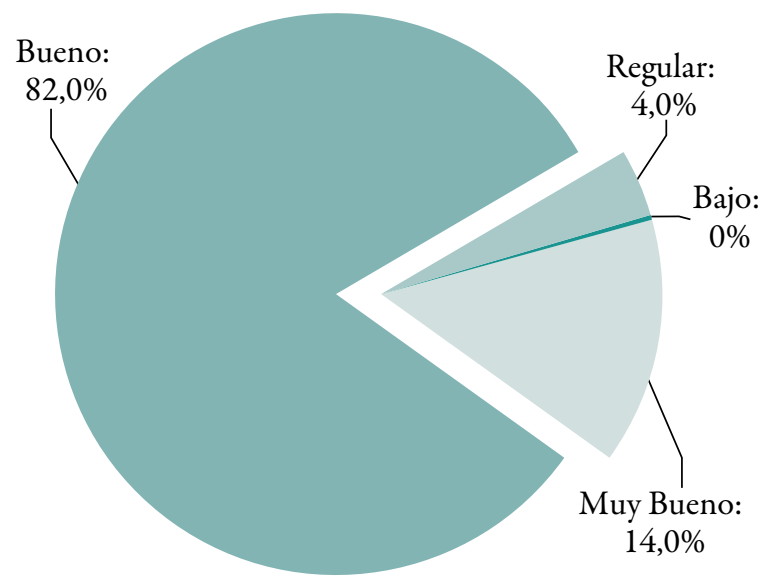

Fuente: elaboración propia (proveniente de tabla 2).

Figura 5. Nivel de rasgos de liderazgo de alumnos de IX y X ciclo de la EAPIC-UAP-Filial Piura (a nivel individual).

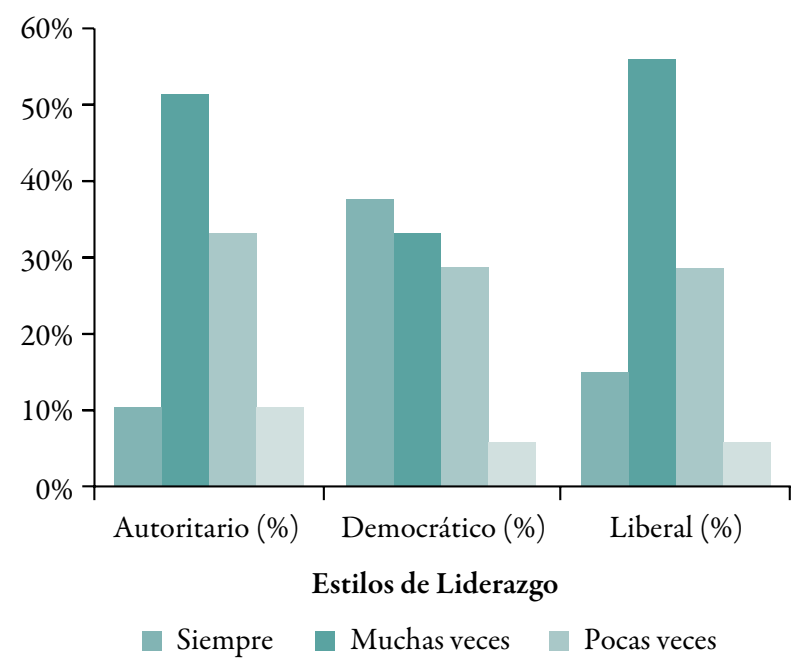

Fuente: elaboración propia (resultados de encuesta a alumnos).

Figura 6. Identificación con estilos de liderazgo a nivel grupal.

Interpretación. De la figura 2 se observa que a nivel grupal las tendencias de liderazgo presentan diversas manifestaciones. Así, el estilo democrático es el más representativo al presentar un mayor porcentaje de alumnos que siempre se identifican con este estilo (36\%); sin embargo, a nivel de identificación general se ubican en primer lugar los estilos democrático y liberal, los cuales tienen un nivel de identificación acumulado de 68\%. En tercer lugar tenemos al estilo autoritario, con una aceptación del 59\% de los alumnos.

Tabla 3. Estilo de trabajo en equipo predominante a nivel grupal, de los alumnos de IX y X ciclo de la EAPIC-UAP-Filial Piura, del ciclo académico 2009-2A.

\begin{tabular}{|c|c|c|c}
\hline ESTILO & $\begin{array}{c}\text { PUNTAJE } \\
\text { OBTENIDO }\end{array}$ & $\begin{array}{c}\text { PORCENTAJE } \\
\text { PARCIAL }\end{array}$ & $\begin{array}{c}\text { PORCENTAJE } \\
\text { ACUMULADO }\end{array}$ \\
\hline Cooperativo & 103 & $22 \%$ & $22 \%$ \\
\hline Impulsador & 96 & $20 \%$ & $43 \%$ \\
\hline Coordinador & 97 & $21 \%$ & $41 \%$ \\
\hline Culminador & 85 & $18 \%$ & $39 \%$ \\
\hline Proveedor & 87 & $19 \%$ & $37 \%$ \\
\hline Total & 468 & $100 \%$ & $100 \%$ \\
\hline
\end{tabular}

Fuente: elaboración propia (resultado de encuestas a alumnos).w

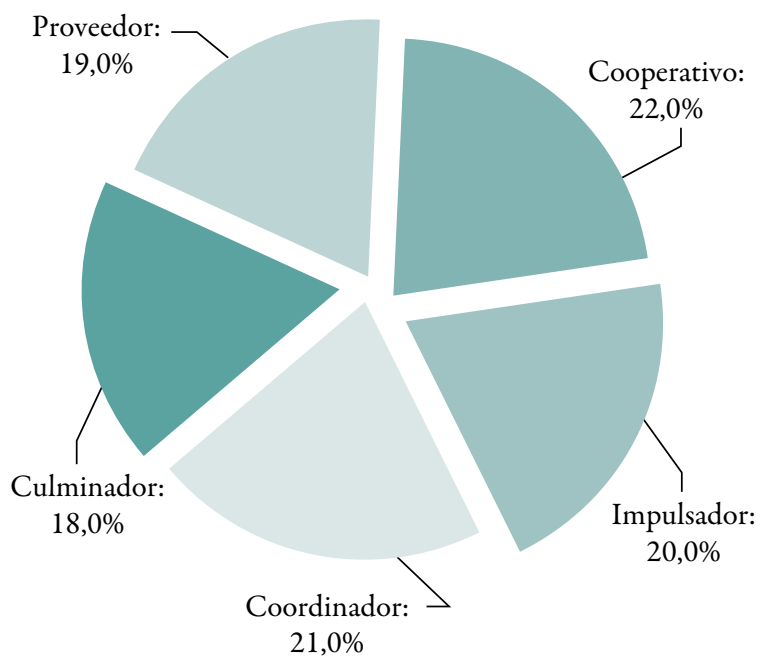

Fuente: elaboración propia (proveniente de tabla 3).

Figura 7. Estilo de trabajo en equipo predominante a nivel grupal de los alumnos de IX y X ciclo de la EAPIC-UAP-Filial Piura, del ciclo académico 2009-2A.

Interpretación. De la tabla 3, obtenemos que el estilo predominante de trabajo en equipo es el estilo cooperativo con un $22 \%$, siguiéndole 
el estilo coordinador con un $21 \%$, el impulsador con un 20\%, el proveedor con un $19 \%$ y finalmente el culminador con un $18 \%$.

Tabla 4. Estilo de manejo de conflictos a nivel grupal, de los alumnos de IX y X ciclo de la EAPIC-UAP-Filial Piura.

\begin{tabular}{|c|c|c|c|}
\hline ESTILO & $\begin{array}{c}\text { PUNTAJE } \\
\text { OBTENIDO }\end{array}$ & $\begin{array}{c}\text { PORCENTAJE } \\
\text { PARCIAL }\end{array}$ & $\begin{array}{c}\text { PORCENTAJE } \\
\text { ACUMULADO }\end{array}$ \\
\hline Dominante & 81 & $20 \%$ & $20 \%$ \\
\hline Integrador & 96 & $24 \%$ & $44 \%$ \\
\hline Servicial & 88 & $21 \%$ & $65 \%$ \\
\hline De evitación & 57 & $14 \%$ & $79 \%$ \\
\hline Negociador & 85 & $21 \%$ & $100 \%$ \\
\hline Total & 406 & $100 \%$ & $100 \%$ \\
\hline
\end{tabular}

Fuente: elaboración propia (resultado de encuestas a alumnos).

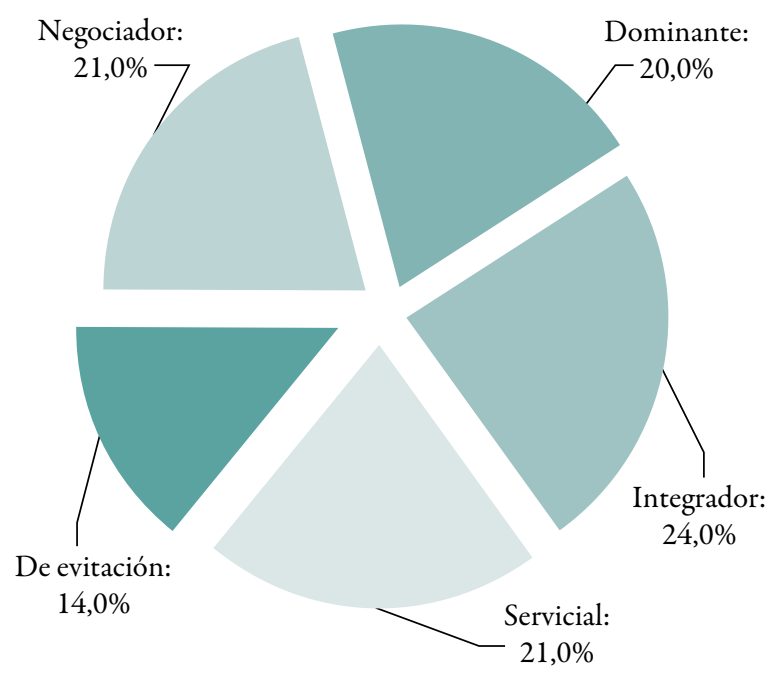

Fuente: elaboración propia (proveniente de tabla 4).

Figura 8. Estilo de manejo de conflictos a nivel grupal, de los alumnos de IX y X ciclo de la EAPICUAP-Filial Piura, del ciclo académico 2009-2A.

Interpretación. De la tabla 4, tenemos que a nivel grupal predomina como estilo de manejo de conflicto el estilo integrador con un $24 \%$ de peso, siguiéndole los estilos negociador y servicial con $21 \%$ cada uno, posteriormente el estilo dominante con un 20\%, y finalmente el de evitación con un $14 \%$.

\section{CONCLUSIONES}

En base a los resultados, podemos indicar lo siguiente respecto a los alumnos de IX y X ciclo de la EAPIC-UAP Filial Piura:

\section{A. Rasgos de liderazgo}

- A nivel general tenemos que el $100 \%$ de los alumnos posee rasgos de liderazgo; sin embargo, al analizar los resultados a nivel individual encontramos que el porcentaje de alumnos que realmente cumple con todos los rasgos de líderes es igual a $96 \%$, de los cuales el $14 \%$ se proyectan como muy buenos líderes, mientras que el otro $4 \%$ no cumple con los rasgos de líderes, lo que permite concluir que el $86 \%$ de nuestros alumnos deberá reforzar los rasgos que no domina a fin de garantizar su competitividad en el mercado laboral.

\section{B. Estilo de trabajo en equipo}

- Encontramos que el 100\% de los alumnos expresa tener capacidad para trabajar en equipo. En paralelo, tenemos que el 59\% de los alumnos maneja alguna herramienta de organización, mientras que el otro $41 \%$ no.

- El 95\% de los alumnos responde al estilo de trabajo tipo impulsador.

- El 100\% de los alumnos responde al estilo de trabajo tipo cooperativo.

- El 100\% de los alumnos responde al estilo de trabajo tipo coordinador.

- El 87\% de los alumnos responde al estilo de trabajo tipo proveedor.

- El 77\% de los alumnos responde al estilo de trabajo tipo culminador.

- Los resultados obtenidos brindan un panorama optimista respecto al desenvolvimiento que proyectarán nuestros futuros egresados, dado que formarán parte de equipos de trabajo que muchas veces se caracterizarán por ser multidisciplinarios, 
por lo que podrán adaptarse adecuadamente ya que presentan un perfil que responde a diversos estilos de trabajo en equipo.

C. Estilo de manejo de conflictos

- El 93\% de los alumnos responde al estilo tipo integrador.

- El 80\% de los alumnos responde al estilo tipo negociador.

- El 85\% de los alumnos responde al estilo tipo servicial.

- El 73\% de los alumnos responde al estilo tipo dominante.

- El 32\% de los alumnos responde al estilo tipo evitador.

\section{Nivel de desempeño laboral}

Finalmente, de los resultados obtenidos en las tres variables, podemos concluir que, desde el enfoque de habilidades organizacionales, nuestros alumnos de IX y X ciclo de la EAPICUAP Filial Piura proyectarán un adecuado nivel de desempeño laboral, de los cuales:

- El 14\% proyectará un alto nivel de desempeño laboral.

- El $86 \%$ proyectará un nivel medio de desempeño laboral.

\section{BIBLIOGRAFÍA}

1. INSTITUTO NACIONAL DE ESTADÍSTICA E INFORMÁTICA (INEI) (2008). Perú: Compendio Estadistico 2008. Lima, Editorial INEI.

2. CHIAVENATO,I.(2004).Comportamiento organizacional: la dinámica del éxito en las organizaciones. México, Editorial Thomson Internacional.

3. FARRO, F. (2005). Evaluación y acreditación para universidades de calidad. Lima, Editorial Udegraf.
4. ROBBINS, S. (2004). Comportamiento organizacional. México, Editorial Pearson Educación.

5. SCHERMERHORN, J; HUNT, J.; OSBORN, R. (2004). Comportamiento organizacional. México D.F, Editorial Limusa.

6. KINICKI, A. y KREITNER, R. (2003). Comportamiento organizacional: conceptos, problemas y prácticas. España, Editorial McGraw-hill.

7. GONZALES, F. (2002). Manual para una eficiente dirección de proyectos y obras. Madrid, Editorial FC.

8. GARCÍA, M., HIERRO, E. y JIMÉNEZ, J. (2001). Selección de personal, sistema integrado. Madrid, Editorial ESIC.

9. FISCHMAN,D.(2000).El camino dellider. Historias ancestrales y vivencias personales. Lima, Editorial UPC, El Comercio.

10. CONSEJO DE EVALUACIÓN, ACREDITACIÓN Y CERTIFICACIÓN DE LA EDUCACIÓN SUPERIOR UNIVERSITARIA. (2008). Modelo de calidad para la acreditación de carreras profesionales universitarias. Lima, CONEAU.

11. FARRO, F. (2007). Guia de organización $y$ presentación de informes de investigación o tesis. Lima, Universidad Alas Peruanas.

12. FARRO, F. (2007). Guia para la elaboración del plan de tesis. Lima, Universidad Alas Peruanas.

13. DIRECCIÓN GENERAL DE INVESTIGACIÓNYACREDITACIÓN UNIVERSITARIA - ANR. (2005). Modelo de autoevaluación con fines de acreditación de las carreras universitarias. Lima, ANR.

14. ANR. (2007). Modelo de autoevaluación con fines de mejora de las carreras universitarias. 
Dirección General de Investigación y Calidad Universitaria.

15. MIYASHIRO, I. (2004). Casos peruanos de comportamiento organizacional. Tomo I (Apuntes de estudio). Lima, Editorial Universidad del Pacífico.

16. ÁVILA, H. (2006). Libro electrónico. Introducción a la metodología de la investigación. Edición electrónica. (www. eumed.net/libros/2006c/203/)

17. HEDBERG, T. (2001). The Role of the Global Engineer: A European View (Educación del ingeniero para el siglo XXI: las actas del 3er. Taller - WWW.books.google.com.pe).

18. ASALDE, J. (2008). Tesis de grado. Grado de coherencia interna entre el perfil propuesto y el plan curricular de la Escuela Profesional de Ingeniería Civil de la Universidad Alas Peruanas, Filial Piura. Piura, Universidad Alas Peruanas.

19. MOSCOL, P. (2008). Tesis de grado. Influencia de la globalización en la creación de valores en las empresas del sector industrial que cotizaron en la Bolsa de Valores de Lima durante el año 2006. Piura, Universidad Alas Peruanas.

20. NAVARRO, A. (2008). Tesis de grado. Diagnóstico de la calidad para la acreditación del Programa de Ingeniería Civil de la UAP. Lima, Universidad Alas Peruanas.

21. PAREDES, M. (2007). Tesis de grado. Propuesta de modelo de comportamiento organizacional sistémico para mejorar la imagen académica de la Facultad de Ciencias Aplicadas de la Universidad Nacional del Centro del Perú. Huancayo, Universidad Alas Peruanas.

22. MONTILlA, I. (2003). Tesis de grado. La calidad de la enseñanza universitaria. Caso: Departamento de Ciencias Económicas y Administrativas, Universidad de los Andes, Núcleo Trujillo. Tarragona, Universitat Rovira i Virgili.
23. SAGOT, C. (1994). Tesis de maestría. Comportamiento gerencial y cambio organizacional: el caso de la C.C.S.S. Universidad de Costa Rica. San José. Editorial UCR, Costa Rica.

\section{PÁGINAS WEBS / LINKS}

- http://www.ucu.edu.uy/CentroPostgrados/ maestrias/ciencias_humanas/Cambio\%20 Organizacional.htm

- http://sisbib.unmsm.edu.pe/BibVirtualData/ Tesis

- http://www.tesisenred.net/TDX-0803104094631/index_cs.html

- http://www.ucab.edu.ve/ucabnuevo/index. php?seccion $=2500 \#$ postgrado

- h t t p://www.rrppet.com.ar/ comportamientoorganizacional.htm

- http://200.16.86.50/digital/MBA/MBAAgotegaray.pdf

- http://www.wgsr.uw.edu.pl/pub/uploads/ actas04/03-bonasewicz.pdf

- http://www.comunidadandina.org/prensa/ discursos/integracionyglobalizacion.pdf

- http://eumed.net/cursecon/ecolat/mx/ADBSPGM.pdf

- http://cybertesis.upc.edu.pe/upc/2004/ garcia_ua/html/index.html

- http://bvs.sld.cu/revistas/aci/vol11_6_03/ aci10603.htm

- http://www.mitecnologico.com/Main/ EstilosDeTrabajoEnEquipo

- http://www.coit.es/publicaciones/bit/ bit172/68-70.pdf

- http://pedagogia-profesional.idoneos. com/index.php/Bibliograf\%C3\%ADa: Fundamentos_del_dise\%C3\%B 1 o_ curricular_por_competencias_laborales

- http://www.congresoretosyexpectativas.udg.mx/ Congreso\%206/Eje\%202/Ponencia_212.pdf

- http://www.anuies.mx/servicios/p_anuies/ publicaciones/revsup/res085/txt10.htm\#3 